\title{
El impacto de la deuda externa pública sobre la inversión privada en México: un análisis de cointegración
}

\author{
Ramón A. Castillo Ponce* \\ Erika García Meneses*
}

\begin{abstract}
Resumen. Existe una relación de desplazamiento entre variables de política fiscal y el gasto privado. Se argumenta, por ejemplo, que el gasto público desincentiva a la inversión privada. Sin embargo, la evidencia formal indica que esta relación de desplazamiento no siempre se cumple. En particular, se ha mostrado que es posible encontrar una relación de complementariedad entre el gasto público y variables reales del sector privado, como el consumo y la inversión, en el largo plazo. En este trabajo analizamos la dinámica estocástica de corto y de largo plazo de la inversión privada y la deuda externa pública en México, una variable que ha sido poco utilizada como proxy del gasto público. Por medio de la estimación de un modelo de corrección de error, derivamos resultados consistentes con los encontrados en la literatura reciente. Esto es, identificamos que, mientras que la deuda pública externa exhibe una relación inversa con la inversión privada en el corto plazo, esta relación se invierte en horizontes amplios.

Palabras clave: deuda externa pública, inversión privada, gasto público.
\end{abstract}

\begin{abstract}
A bstract. Evidence suggests that the implementation of public policy crowds out private expenditure. It is argued, for example, that public spending inhibits private investment. However, formal evidence indicates that this crowding out effect does not always hold. In particular, it has been shown that it is possible to find a complementary relation between public spending and real variables of the private sector such as consumption and investment in the long run. In this document we analyze the short run and long run stochastic dynamics of private investment and foreign public debt in Mexico, a variable that has been scarcely used as a proxy for public spending. With the estimation of an error correction model we derive results that are consistent with what is found in the literature. That is, we identify that, while foreign public debt exhibits a negative relation with private investment in the short run, the relationship becomes positive for longer horizons. Keywords: foreign public debt, private investment, public spending.
\end{abstract}

* Facultad deEconomía y Relaciones Internacionales dela Universidad A utónoma de Baja California.

Correos electrónicos: rcastil@calstatela.edu y amerika101284@yahoo.com.mx 


\section{Introducción}

La relación entre el ejercicio del gasto público y el comportamiento de variables del sector real ha preocupado tanto a tomadores de decisiones como a académicos durante décadas. ${ }^{1}$ La importancia de dicha relación es evidente: si la instrumentación de política económica presenta un efecto significativo sobre variables del sector privado, entonces le es posible a la autoridad pública influir sobre el desempeño de la economía.

Una de las asociaciones más estudiadas en la ciencia económica es la que existe entre el gasto público y la inversión privada. Tradicionalmente, el efecto de las políticas fiscales sobre la inversión privada se ha llevado a cabo considerando a la inversión pública como la variable de control. Dentro de esta perspectiva encontramos varios estudios que han contribuido significativamente a la literatura relacionada con el tema. Blejer y Khan (1984), por ejemplo, encuentran evidencia de desplazamiento o crowding out entre la inversión pública y la privada en un panel de 24 países en desarrollo, dentro de los que se incluye a México. Rama (1993), por su parte, realiza un compendio de estimaciones empíricas de la función de inversión en países subdesarrollados y encuentra, igualmente, la presencia de un efecto de crowding out de la inversión pública sobre la privada. Sin embargo, Servén y Solimano (1993), en un análisis de tipo cross-country de 15 países subdesarrollados (incluyendo a México), encuentran que predomina el efecto de complementariedad o crowding in. A resultados similares llega Cardoso (1993), quien aplica un análisis de panel a una muestra de siete países para el periodo 1970-1985, y encuentra que existe crowding in de la inversión pública hacia la privada.

Para el caso de México, es escaso el número de trabajos de investigación que se han realizado sobre el tema. Cabe mencionar a Aschauer y Lachler (1998), quienes realizan un estudio de series de tiempo en el cual encuentran que la inversión pública desplazó a la inversión pri-

${ }^{1}$ La literatura sobre el tema es amplia, aquí nos podemos referir por ejemplo a Barro (1984), Aschauer (1989) y más recientemente Giavazzi et al. (2002). 
vada durante el periodo 1970-1996. También Herrera (2003) identifica una relación significativa en el largo plazo de crowding out entre la inversión privada y la pública; resultado contrario al que encuentran Castillo y Herrera (2005), quienes muestran que aunque en el corto plazo la inversión pública desplaza a la inversión privada, dicha relación se invierte en el largo plazo. Esto es, la inversión pública complementa a la inversión privada cuando se consideran horizontes amplios.

Nótese que, a pesar de que el tema de la relación entre el gasto público y la inversión privada ha sido ampliamente analizado, a la fecha no se encuentra un consenso en cuanto a la relación que existe, $o$ debe existir, entre ambos rubros. Esto es, mientras que algunos estudios identifican una relación de desplazamiento, otros muestran que el gasto público puede incentivar el gasto privado. En el presente análisis intentamos contribuir al debate tomando un acercamiento poco explorado en la literatura existente. En particular, consideramos a la deuda pública externa, y no al gasto público, como la variable que describe el comportamiento del sector público. Naturalmente, el presente no es el primer documento que relaciona a estas dos variables. Borensztein (1990), por ejemplo, prueba la hipótesis de que el sobreendeudamiento externo desincentiva a la inversión privada. Asimismo, Cohen (1993) examina la incipiente inversión asociada con el alto nivel de deuda de países en desarrollo durante la década de los ochenta. Sus resultados indican que el servicio de la deuda desplaza a la inversión, entre otras razones a causa de la competencia que el sector público realiza con la empresa privada para conseguir medios de financiar la deuda. Más recientemente, Hansen (2002) encuentra que una disminución de la ayuda oficial en el mismo monto que un aumento simultáneo en el alivio de la carga de la deuda (servicio) no tiene efectos en el crecimiento, mientras que puede llevar a una caída de las tasas de inversión. ${ }^{2}$

Cabe mencionar que aunque en general los resultados reportados en estudios anteriores apuntan a una relación inversa entre el endeu-

${ }^{2}$ Otros documentos que evalúan la relación entre deuda pública externa e inversión privada incluyen a Elbadawi et al. (1997), Were (2001), y Serieux y Samy (2001). 
damiento público y la inversión privada, la misma no es contundente. Podemos mencionar, por ejemplo, un estudio para Colombia en el que se encuentra que la deuda externa no presenta un efecto significativo sobre la inversión privada. ${ }^{3}$ Ribeiro y Teixeira (2001) encuentran también que al incluir la variable de deuda externa en un modelo de determinación de la inversión, el gasto público resulta no significativo. Más aún, como ya se indicó, algunos análisis sobre la relación entre el gasto público y la inversión privada en México presentan resultados encontrados. Así, aunque en principio podríamos anticipar una relación negativa entre el endeudamiento público externo y la inversión privada, no descartamos la posibilidad de que los dos rubros se complementen en el largo plazo.

La herramienta que instrumentamos en nuestro análisis empírico se refiere a un Modelo de Corrección de Error (MCE), el cual nos permite identificar la existencia de cointegración entre la deuda pública externa y la inversión privada; así como determinar su dinámica en el corto plazo. Creemos que es importante distinguir entre el efecto de corto plazo y el de largo plazo, ya que la relación cualitativa puede cambiar dependiendo del horizonte relevante.

El análisis que continúa se estructura en cuatro apartados. Además de esta introducción, en el segundo apartado presentamos una descripción del comportamiento de la deuda externa pública en México, así como su relación con la inversión privada. El propósito de esta sección es ilustrar de manera gráfica la relación que han mantenido ambos rubros a través del tiempo. Esta relación es luego establecida formalmente con la instrumentación del ejercicio econométrico que se lleva a cabo en el tercer apartado, el cual considera, como ya se indicó, la estimación de un MCE que nos permite identificar las dinámicas de largo y de corto plazos entre las variables de interés. En esta sección se describen los datos y se realizan las pruebas estadísticas pertinentes para la estimación del modelo. El cuarto y último apartado concluye indicando algunas implicaciones de los resultados respecto al quehacer de las políticas públicas.

\footnotetext{
${ }^{3}$ Véase Rubio et al. (2003).
} 


\section{Deuda pública externa como fuente de financiamiento ${ }^{4}$}

La deuda externa de México es un tema que se ha tratado abundantemente en la literatura, ${ }^{5}$ de modo que nuestro propósito en este apartado se acota a presentar una descripción general de su comportamiento y a ilustrar gráficamente su relación con la inversión privada. Elaboramos sobre el primer objetivo al inicio del apartado destacando el uso que se le ha dado a la deuda externa a través del tiempo. En la segunda parte mostramos el comportamiento de la deuda y la inversión privada a partir de 1993, notando la posible relación estadística que pudiéramos encontrar entre ellas.

\section{Naturaleza de la deuda pública externa}

La deuda pública externa considera todos los créditos contratados por el sector público en forma directa o a través de sus agentes financieros con entidades financieras del exterior y pagaderos en el extranjero en moneda diferente a la moneda nacional. ${ }^{6}$ Algunos de los factores que intervienen en la integración de la deuda pública externa incluyen colocaciones públicas en los mercados internacionales de capital, financiamientos de los organismos financieros internacionales y la deuda estructurada. ${ }^{7}$ En el cuadro 1 se presenta una descripción del crecimiento de la deuda pública externa en diferentes periodos de la historia contemporánea de México.

${ }^{4}$ Borensztein et al. (2006) presentan una excelente caracterización del uso de la deuda pública como medio de financiamiento del gasto público.

${ }^{5}$ Para una amplia descripción del comportamiento de la deuda externa de México véase Green (1987). Para información oficial sobre los niveles de deuda externa pública que ha mantenido México se pueden revisar los informes anuales del Banco de México.

${ }^{6}$ Para propósitos del presente estudio, no distinguimos entre deuda contratada con organismos internacionales y aquella contratada con la banca internacional comercial. Creemos que esta distinción no influye sobre los resultados que obtenemos.

${ }^{7}$ Deuda pública como la define la Secretaría de Hacienda y Crédito Público (SHCP). 
Cuadro 1.

Crecimiento de la deuda pública externa

\begin{tabular}{cc}
\hline Periodo & Crecimiento $(\%)^{*}$ \\
\hline $1946-1955$ & 26.80 \\
$1955-1972$ & 16.00 \\
$1973-1982$ & 28.00 \\
\hline
\end{tabular}

Fuente: Deuda externa pública mexicana, SHCP (1998).

*Tasa de crecimiento promedio anual.

Notamos que la deuda externa pública mantuvo un crecimiento sostenido de 1946 a 1982. De acuerdo a información proporcionada por la SHCP, los recursos obtenidos se destinaron principalmente a la inversión física. Es decir, el gobierno utilizó los fondos para el financiamiento de la inversión en empresas paraestatales y el fortalecimiento de la banca de desarrollo del sector público.

En el cuadro 2 se presenta la distribución de recursos en el sector público para los subperiodos 1972-1976 y 1977-1982. Destaca que durante el primer subperiodo, el gobierno federal, Pemex, el sector eléctrico y Nafinsa obtuvieron las mayores proporciones de recursos. De

Cuadro 2.

Distribución de la deuda pública externa

\begin{tabular}{lrrcc}
\hline \multirow{2}{*}{ Entidad } & \multicolumn{2}{c}{ Periodo } & 1972-1976 & \multicolumn{2}{c}{ Periodo $1977-1982$} \\
& mMDls. & $\%$ & mMDls. & $\%$ \\
\hline Total & 15.054 & 100 & 39.274 & 100 \\
Gob. federal & 2.122 & 14.1 & 5.274 & 14.3 \\
Pemex & 1.713 & 11.4 & 13.733 & 35 \\
Sect. eléctrico & 2.208 & 14.7 & 2.984 & 7.5 \\
Nafinsa & 2.198 & 14.6 & 7.85 & 20 \\
Banobras & 0.578 & 3.8 & 4.217 & 10.7 \\
Banrural & 0.576 & 3.8 & 1.723 & 4.4 \\
Otros & 5.659 & 37.6 & 3.148 & 8 \\
\hline
\end{tabular}

Fuente: Deuda externa pública mexicana, SHCP (1988). 


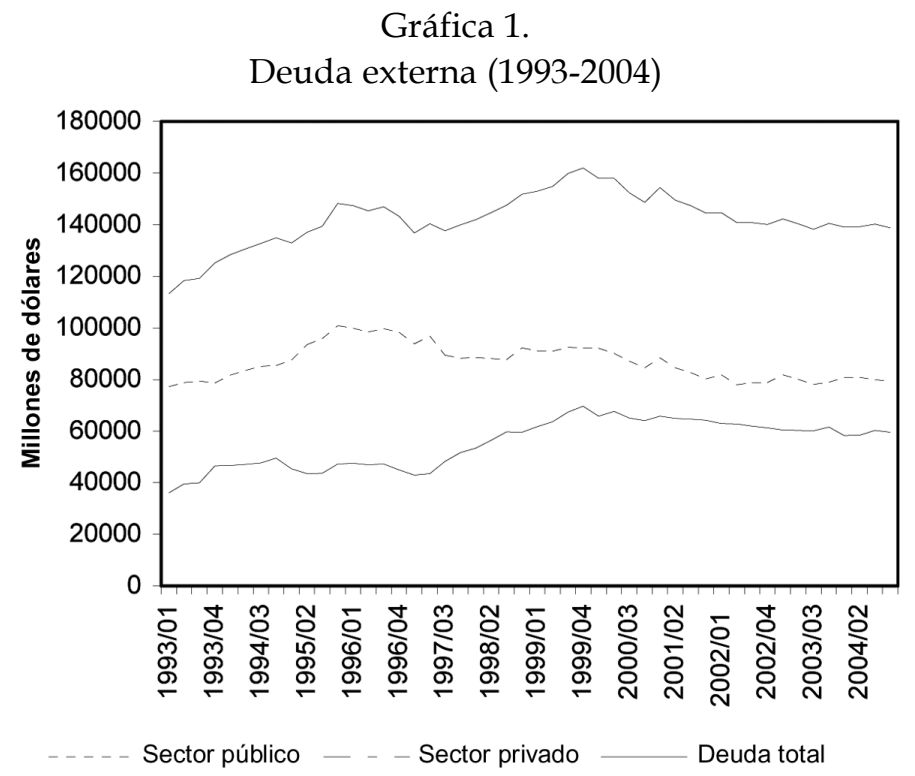

1977 a 1982, sin embargo, Banobras sobrepasó al sector eléctrico en la proporción de recursos recibidos. Asimismo, notamos que en este último periodo el sector petrolero fue el más beneficiado, dato no sorprendente si consideramos que para entonces México se convirtió en el cuarto país productor de petróleo a nivel mundial. La utilización de los recursos en Pemex se reflejó en la conclusión de La Cangrejera, el complejo petroquímico más grande del mundo.

La práctica de financiarse con recursos externos, sin embargo, cambió a partir de mediados de los ochenta. El país entró en un periodo de desestabilización económica que obligó al sector público a disciplinar su gasto, y en consecuencia los préstamos provenientes del exterior disminuyeron. Como se muestra en la gráfica 1, la deuda pública externa presenta una tendencia no creciente en los últimos años.

La deuda pública externa y la inversión privada

Como indicamos anteriormente, en general se señala que el propósito por el cual algunos países recurren al mercado internacional de fon- 
dos es el financiamiento de ciertas actividades que pueden resultar en crecimiento económico. En el caso de México, el argumento tradicional se refirió a la necesidad de fortalecer la industria nacional. Haciendo un poco de historia, recordamos que a partir de la década de los cincuenta y hasta principios de los ochenta el Modelo de Sustitución de Importaciones (MSI) predominó en el país. La idea era fortalecer la economía interna a través de la sustitución de bienes de consumo, bienes intermedios y bienes de capital que provenían del exterior, por producción interna. Así, durante este periodo, al menos en teoría, los recursos externos se invirtieron fundamentalmente en el desarrollo de infraestructura y en el financiamiento de programas de incentivos para la industria doméstica. ${ }^{8}$

En 1979 se dio el boom petrolero, por lo que el endeudamiento externo se canalizó al desarrollo de esa industria. Lo que sucedió durante los primeros años de los ochenta, y que llevó al país a experimentar una de las más significativas crisis económicas en su historia, se puede resumir en dos ideas. La primera se refiere a la caída de los precios del petróleo en 1981, y la segunda a la falta de rendimientos de las inversiones realizadas bajo el marco del MIs. Así, el alto endeudamiento y la escasez de ingresos llevó a que en 1982 México declarara una moratoria en el pago de sus obligaciones externas. En palabras de Gavito y Reyes (1990), "la deuda contratada sirvió para financiar el consumo, inversión de escasa rentabilidad y fugas de capital, gastos que en alguna medida reflejaron errores de la política económica y que mermaron la capacidad de pago de las economías endeudadas". Cabe señalar también que la facilidad para endeudarse en este periodo respondió en parte a la liquidez que se presentó en el mercado de eurodólares, y que permitió que la banca comercial transnacional prestara importantes sumas de capital sin contar con aval gubernamental. Asimismo, viéndolo en retrospectiva, notamos que a Estados Unidos le favoreció el desarrollo de infraestructura petrolera en México, ya que le permitió adquirir reservas estratégicas a cambio de compras anticipadas. $^{9}$

\footnotetext{
${ }^{8}$ Estos incentivos incluyeron préstamos y subsidios, entre otros.

${ }^{9}$ Véase Girón (1991).
} 
El financiamiento de las actividades productivas durante las décadas de los ochenta y noventa provino mayormente de recursos del sector privado a través del programa de privatización que impulsó el gobierno federal. Destacamos que esta transición de deuda externa a deuda interna no necesariamente fue el resultado de una decisión propia de las autoridades mexicanas, sino que se dio en respuesta a la estrategia que dictaran las instituciones que capitalizaron México durante la crisis de deuda de los ochenta, de tal suerte que durante este periodo encontramos las renegociaciones de la deuda mexicana con la banca privada internacional y la implementación del Plan Brady. Tomando en cuenta estos acontecimientos, no resulta sorprendente que la deuda externa pública se haya mantenido en niveles razonables en los últimos años. ${ }^{10}$

En la gráfica 2 se muestra la relación entre la tasa de crecimiento de la deuda pública externa y la inversión privada para el periodo 19932004.

\section{Gráfica 2.}

Evolución de la deuda pública externa y la inversión privada

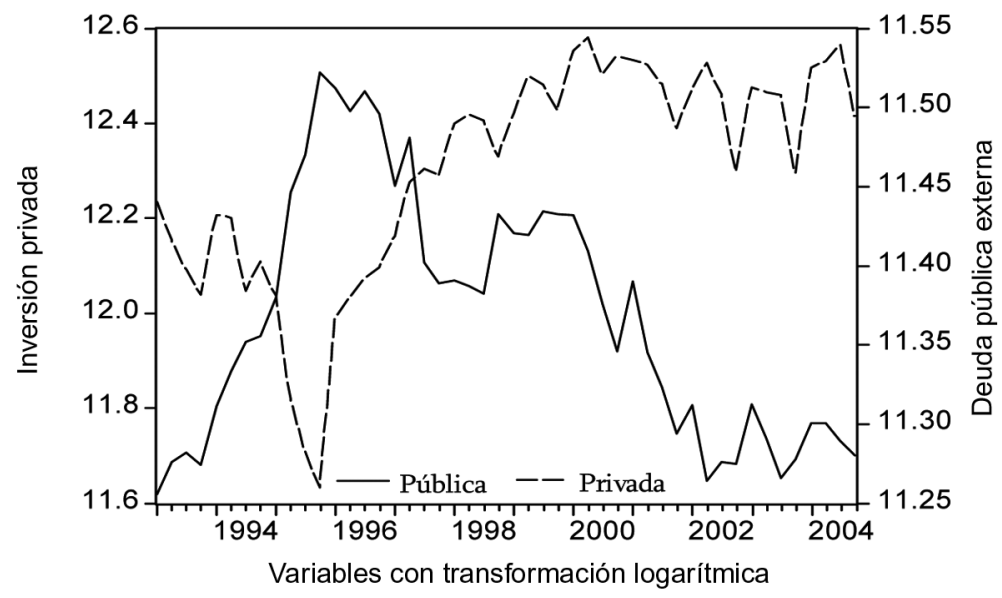

\footnotetext{
${ }^{10}$ Para una descripción amplia de la crisis de deuda que enfrentó América Latina véase Franko (2006) o Lustig (1997) para el caso particular de México.
} 
Como se puede observar, de 1993 a 1997 la inversión privada guarda una aparente relación negativa con la deuda pública externa. Notamos que la misma puede ser el resultado de la caída en la inversión privada durante la crisis económica de 1994. A partir de 1998, la relación entre las variables ya no es obvia, pues mientras que se evidencia una desaceleración importante en la tasa de crecimiento de la deuda externa, la inversión privada parece crecer a una tasa constante.

\section{Ejercicio empírico}

Nuestro propósito en este apartado es establecer formalmente la relación estocástica que existe entre la deuda externa y la inversión priva en México. Con este fin, tomamos datos publicados por fuentes oficiales y los sometemos a un análisis econométrico riguroso. El mismo incluye una evaluación de la naturaleza estocástica de las series y la estimación de un modelo de corrección de error. La elección de este modelo en particular responde al hecho de que nos es posible estimar relaciones estables de corto y de largo plazos, de tal forma que a través de los resultados obtenidos podremos identificar los efectos transitorio y permanente que presenta la deuda externa pública sobre la inversión privada.

\section{Datos}

El periodo de estudio se extiende desde el primer trimestre de $1993 \mathrm{al}$ cuarto trimestre de 2004. Las variables de interés se refieren a la deuda pública externa, la inversión privada y el Producto Interno Bruto (PIB) como variable de control. La fuente de los datos es el Instituto Nacional de Estadística, Geografía e Informática (INEGI), la Secretaria de Hacienda y Crédito Público (SHCP) y el Banco de México (Banxico). Las variables se transforman logarítmicamente y se denotan como sigue:

- Deuda pública externa (ldepu)

- Inversión privada (linopr)

- PIB (lpib) 


\section{Naturaleza estocástica de las series}

En este ejercicio nos interesa evaluar la relación que mantienen las variables descritas anteriormente en el corto y en el largo plazos. Para tal propósito la metodología apropiada se refiere a la teoría de la cointegración. Como es ampliamente conocido, para instrumentar dicho análisis es necesario que las series sean no estacionarias e integradas de orden uno, I (1). Para verificar esta propiedad se efectuó la prueba de raíz unitaria sugerida por Dickey y Fuller (1979) en su forma aumentada (ADF). La misma considera la hipótesis nula de no estacionariedad de la serie contra la alternativa de estacionariedad. Los resultados se resumen en el cuadro 3. Nótese que en todos los casos la hipótesis nula no se rechaza en niveles pero sí en primeras diferencias, lo cual indica que las series se encuentran integradas de orden 1.

\section{Estimación}

Para estimar las relaciones de corto y de largo plazos entre las variables de interés se instrumenta un MCE, mismo que se deriva a partir de un modelo autorregresivo de rezagos distribuidos (ARD).

Cuadro 3.

Pruebas de raíz unitaria

\begin{tabular}{llccc}
\hline \multicolumn{5}{c}{ Prueba Dickey Fuller (ADF) } \\
\hline \multirow{2}{*}{ Variable } & \multicolumn{4}{c}{ Tendencia e intercepto } \\
Nivel & 1ra diferencia & $\begin{array}{c}\text { Orden de } \\
\text { Integración }\end{array}$ \\
\hline \multirow{2}{*}{ Invpro } & Estadístico & -2.51 & -5.04 & $\mathrm{I}(1)$ \\
& Valor crítico & -3.5 & -3.51 & \\
Ipib & Estadístico & -3.15 & -4.33 & $\mathrm{I}(1)$ \\
& Valor crítico & -3.51 & -3.53 & $\mathrm{I}(1)$ \\
& Estadístico & -2.29 & -7.07 & \\
& Valor crítico & -3.5 & -3.51 & \\
\hline
\end{tabular}


Suponemos que el ARD se representa de la siguiente forma:

$$
y_{t}=m+\alpha_{1} y_{t-1}+\beta_{0} x_{t}+\beta_{1} x_{t-1}+\varepsilon_{t}
$$

Remplazando $y_{1} \operatorname{con} y_{t-1}+\Delta y_{t}$ y $x_{t} \operatorname{con} x_{t-1}+\Delta x_{t}$ en (1) obtenemos

$$
\Delta y_{t}=m+\beta_{0} \Delta x_{t}-(1-\alpha) y_{t-1}+\left(\beta_{0}+\beta_{1}\right) x_{t-1}+\varepsilon_{t}
$$

Que se puede reescribir como

$$
\Delta y_{t}=\beta_{0} \Delta x_{t}-\left(1-\alpha_{1}\right)\left[y_{t-1}-\frac{m}{1-\alpha_{1}}-\left(\frac{\beta_{0}+\beta_{1}}{1-\alpha_{1}}\right) x_{t-1}\right]+\varepsilon_{t}
$$

el cual representa el MCE, donde el término de ajuste es la expresión dentro de los corchetes. Para nuestro ejercicio, reescribimos (2) para un sistema de tres variables:

$$
\begin{aligned}
& \Delta y_{t}=\alpha_{0}+\alpha_{1} y_{t-1}+\alpha_{2} x_{t-1}+\alpha_{3} z_{t-1}+\beta_{1} \Delta y_{t-1}+\ldots+\beta_{n} \Delta y_{t-n}+ \\
& \varphi_{0} \Delta x_{t}+\varphi_{1} \Delta x_{t-1}+\ldots+\varphi_{k} \Delta x_{t-k}+\gamma_{0} \Delta z_{t}+\gamma_{1} \Delta z_{t-1}+\ldots+ \\
& \gamma_{p} \Delta z_{t-p}+\psi D_{t}+\phi T+\varepsilon_{t}
\end{aligned}
$$

donde $\alpha_{1}$ es la velocidad de ajuste, $\alpha_{2}$ y $\alpha_{3}$ representan las elasticidades de largo plazo, las $\beta^{\prime}$ s suman la dinámica de corto plazo, $D_{t}$ es un vector de variables determinísticas y $T$ es una tendencia. La cointegración se cumple si el coeficiente de ajuste resulta negativo, estadísticamente significativo y menor a 1 en términos absolutos.

Utilizando una metodología de lo general a lo particular, se derivó la siguiente especificación que resultó ser la más eficiente en términos estadísticos. ${ }^{11}$

$$
\begin{aligned}
& \Delta \text { linvpr }_{t}=\alpha_{0}+\alpha_{1} \text { linvpr }_{t-1}+\alpha_{2} \text { lpib }_{t-1}+\alpha_{3} \text { ldepu }_{t-1}+\beta_{0} \Delta l p i b_{t} \\
& +\beta_{4} \Delta l p i b_{t-4}+\delta_{3} l \Delta d e p u_{t-3}+\psi t
\end{aligned}
$$

\footnotetext{
${ }^{11}$ Se realizaron pruebas estadísticas de eficiencia y estabilidad sobre los residuales. Los resultados se presentan en el apéndice.
} 
El cuadro 4 muestra los resultados de la estimación de la ecuación anterior.

\author{
Cuadro 4.
}

Estimación del MCE

Variable dependiente: $\mathrm{d}($ linvpr $)$

\begin{tabular}{|c|c|c|c|c|c|c|c|c|}
\hline & \multirow{2}{*}{$\begin{array}{c}\text { Intercepto } \\
\alpha_{0}\end{array}$} & \multirow{2}{*}{$\begin{array}{c}\text { Coeficiente } \\
\text { de ajuste } \\
\text { linvpr }(-1) \\
\alpha_{1}\end{array}$} & \multicolumn{2}{|c|}{$\begin{array}{c}\text { Dinámica } \\
\text { de largo plazo }\end{array}$} & \multicolumn{4}{|c|}{ Dinámica de corto plazo } \\
\hline & & & $\begin{array}{c}\operatorname{lpib}(-1) \\
\quad \alpha_{2}\end{array}$ & $\begin{array}{c}\text { ldepu }(-1) \\
\alpha_{3}\end{array}$ & $\begin{array}{c}d(l p i b) \\
\beta_{0}\end{array}$ & $\begin{array}{c}d(\operatorname{lpib}(-4)) \\
\beta_{4}\end{array}$ & $\begin{array}{c}d(\operatorname{depu}(-3)) \\
\beta_{3}\end{array}$ & $\begin{array}{c}t \\
\psi\end{array}$ \\
\hline $\begin{array}{l}\text { Estimador } \\
\text { e.s. }\end{array}$ & $\begin{array}{l}-56.85 \\
(9.821)\end{array}$ & $\begin{array}{l}-0.60 \\
(0.108)\end{array}$ & $\begin{array}{c}2.71 \\
(0.488)\end{array}$ & $\begin{array}{c}0.63 \\
(0.18)\end{array}$ & $\begin{array}{c}2.39 \\
(0.452)\end{array}$ & $\begin{array}{c}-1.57 \\
(0.426)\end{array}$ & $\begin{array}{c}-0.93 \\
(0.361)\end{array}$ & $\begin{array}{c}-0.01 \\
(0.002)\end{array}$ \\
\hline t-statistic & -5.788 & -5.529 & 5.559 & 3.531 & 5.296 & -3.683 & -2.577 & -3.876 \\
\hline
\end{tabular}

Nota: Las series están en logaritmos, por lo tanto, los estimadores representan elasticidades, excepto la variable t. La letra d antepuesta a la variable representa que se trata de una serie en diferencias y la variable $t$ es tendencia.

Primero, destacamos el término de ajuste, $\alpha_{1}$, el cual cumple con las características necesarias para sugerir la existencia de cointegración; menor a 1 en valor absoluto, negativo y significativo. Interpretado éste como velocidad de ajuste, su magnitud, 0.60, indica que ante choques transitorios que desvíen el sistema de su estado estacionario (como un cambio en la política económica), el sistema regresará al equilibrio en aproximadamente siete meses, lo cual es relativamente rápido.

Siguiendo con la derivación del MCE, calculamos las elasticidades de largo plazo estimando $\alpha_{2} / \alpha_{1}$ y $\alpha_{3} / \alpha_{1}$ para el Рів y para la deuda pública respectivamente. Encontramos una elasticidad de la inversión privada respecto al producto de 4.515 y de 1.056 para la deuda pública. El primer resultado es consistente con la evidencia encontrada en estudios anteriores. ${ }^{12}$ En particular, notamos que existe una relación positiva entre la inversión privada y el PIB, y que la magnitud de la

12 Véase Herrera (2003), por ejemplo. 
elasticidad es mayor a 1, lo cual indica un efecto acelerador del producto a la inversión. Resulta interesante que la elasticidad de largo plazo de la inversión respecto a la deuda pública sea positiva y cercana a la unidad. Como se mencionó en la sección introductoria, éste es un resultado que se ha obtenido en análisis recientes del efecto del gasto público sobre variables del sector privado. De esta forma, consistente con lo encontrado por Castillo y Herrera (2005), por ejemplo, se identifica una relación de complementariedad entre el ejercicio del gasto público y la inversión privada en México en el largo plazo.

En cuanto a la dinámica de corto plazo, la respuesta de la inversión privada al PIB es positiva y con una magnitud neta de aproximadamente 0.82. Para la deuda pública externa el resultado indica una relación negativa de 0.93 . Esto es, en el corto plazo la inversión privada responde inversamente a incrementos en la deuda.

La gama de explicaciones sobre la respuesta negativa de la inversión a la deuda en el corto plazo y su relación de complementariedad en horizontes largos es amplia. Aquí ofrecemos una que es consistente con principios económicos tradicionales: haciendo un poco de historia, encontramos que en las décadas de los cincuenta, sesenta y setenta México adquirió niveles de endeudamiento significativos debido a la implementación del MSI, el cual no rindió los resultados esperados; las consecuencias de su deterioro fueron evidentes en los años ochenta. Así, la adquisición de deuda externa se convirtió en un indicador de problemas potenciales para la economía. Considerando nuestro periodo de análisis, no es sorprendente encontrar entonces que la respuesta transitoria de la inversión al endeudamiento sea inversa. Sin embargo, es claro que para finales de los noventa el gasto público ejercido en años anteriores había creado la infraestructura necesaria para incentivar la inversión privada. Líneas telefónicas, de electricidad, carreteras y otras obras públicas facilitaron el desarrollo de la inversión privada. De tal forma que, ya considerando un horizonte mayor a diez años (como se hace en este ejercicio), es razonable encontrar que la deuda externa pública, que al menos en parte fue utilizada para financiar trabajos de infraestructura, muestre una relación positiva con la inversión privada. 


\section{Conclusiones}

La dinámica del endeudamiento público externo en México ha sido altamente volátil. A través del tiempo pasó de ser una fuente de financiamiento poco utilizada a representar prácticamente la principal fuente de capital para proyectos de inversión y desarrollo. Entender su influencia sobre las decisiones del sector privado no es un ejercicio trivial. Algunos autores, como Blejer y Khan (1984) por ejemplo, identifican una respuesta negativa de la inversión al ejercicio del gasto público, lo cual es consistente con el clásico argumento de crowding out; otros, como Servén y Solimano (1993), sin embargo, encuentran una interacción de complementariedad, debido a lo cual no es posible argumentar que existe una relación clara o definitiva entre las dos variables.

A diferencia de estudios anteriores, en este trabajo nos dimos a la tarea de identificar la respuesta de corto y de largo plazos de la inversión privada a la deuda externa. Los resultados de la estimación de un MCE muestran que existe una relación negativa entre las dos variables en el corto plazo pero de complementariedad para horizontes amplios. Revisando la literatura existente, identificamos que trabajos similares han encontrado resultados que apuntan en la misma dirección. De reciente publicación, encontramos a Castillo y Herrera (2005), cuyos resultados indican que el gasto público parece desincentivar a la inversión privada sólo transitoriamente.

Es claro, sin embargo, que es necesario continuar con esta línea de investigación para establecer de manera más precisa la importancia del gasto público sobre variables del sector privado. Nótese, por ejemplo, que en este documento no identificamos los canales por medio de los cuales la deuda pública externa incentiva a la inversión privada, sino que nos limitamos a proveer un argumento razonable para explicar su posible interacción. Sería valioso realizar un ejercicio que derive el mecanismo a través del cual el gasto del sector público afecta las decisiones del sector privado. Podríamos, por ejemplo, examinar cómo los cambios en las tasas de interés que resultan de un incremento en el gasto público se relacionan con variaciones en la inversión privada. De igual forma, podríamos pensar en un escenario en el que las deci- 
siones sobre el gasto público cambian las expectativas de inversionistas en el sector privado, en un sentido o en otro. Así, pensamos en este ejercicio como una primera aproximación al estudio de la relación que existe entre la deuda pública externa y la inversión privada. Dejamos la puerta abierta para evaluar posibilidades como las ya mencionadas en investigaciones futuras. 


\section{APÉNDICE}

Cuadro A.

Prueba de normalidad en los residuos

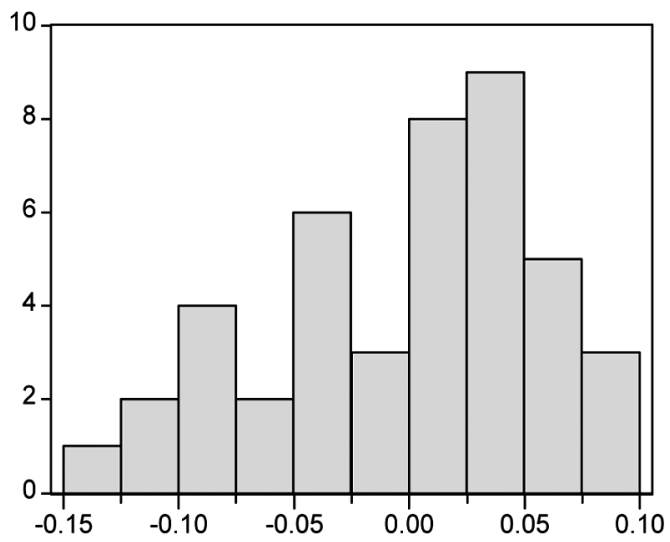

Series: Residuals

Sample 1994:2 2004:4

Observations 43

Mean

5.15E-15

Median

0.021170

Maximum

0.092954

Minimum

$-0.125065$

Std. Dev. $\quad 0.058034$

Skewness $\quad-0.498594$

Kurtosis $\quad 2.182784$

Jarque-Bera $\quad 2.978153$

Probability

0.225581

Cuadro B.

Pruebas de diagnóstico de la regresión del MCE

Regresión

Estadístico P-value

Prueba Reset de Ramsey de Especificación*

F-Statisic

0.156

0.958

Log likelihood ratio

0.858

0.930

Prueba Breusch-Godfrey de Autocorrelación*

F-Statisic

0.229

0.919

Obs* $\mathrm{R}^{2}$

1.237

0.871

Prueba White (no cross terms) de Heteroscedasticidad

$\begin{array}{lll}\text { F-Statisic } & 0.489 & 0.912 \\ \text { Obs* }^{2} & 7.735 & 0.860\end{array}$

Prueba White (cross terms) de Heteroscedasticidad

F-Statisic

$\mathrm{Obs}^{*} \mathrm{R}^{2}$

0.811

0.690

32.181

0.507

Prueba ARCH LM*

F-Statisic

0.307

$\mathrm{Obs}^{*} \mathrm{R}^{2}$

1.362
0.870

0.850

*Pruebas estimadas con cuatro rezagos. 


\section{Cuadro C.}

Prueba de estabilidad de la regresión del MCE.
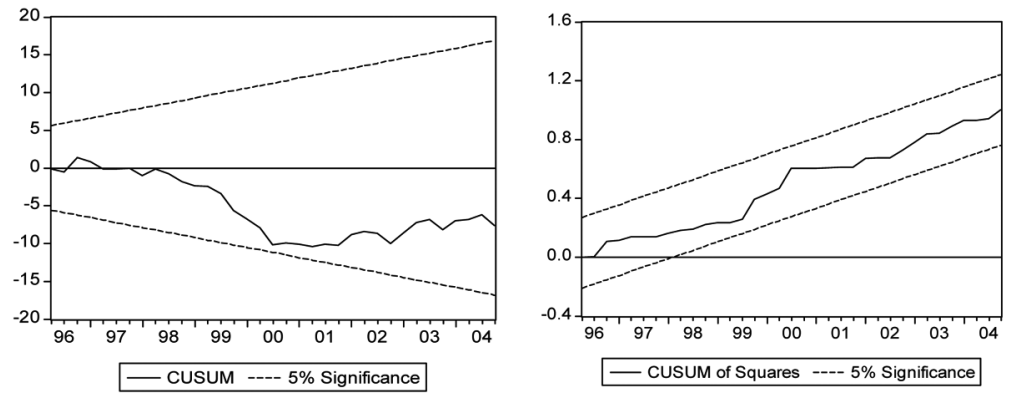


\section{Bibliografía}

Aschauer, David, 1989, "Does Public Capital Crowd Out Private Capital?", Journal of Monetary Economics, 24:171-188.

y Ulrich Lachler, 1998, "Public Investment and Economic Growth in Mexico", Policy Research Working Paper, Banco Mundial.

Banco de México, Informes anuales.

Barro, Robert, 1981, “Output Effects of Government Purchases”, Journal of Political Economy, 89:1086-1121.

Blejer, Mario y Mohsin Khan, 1984, Government Policy and Private Investment in Developing Countries, Fondo Monetario Internacional.

Borensztein, Eduardo, 1990, Debt Overhang, Debt Reduction and Investment: The Case of the Philippines, Fondo Monetario Internacional.

Borensztein, Eduardo, Eduardo Levy y Ugo Panizza, 2006, Living with Debt, How to Limit the Risks of Sovereign Finance, Harvard University Press.

Cardoso, Eliana, 1993, "Macroeconomic Environment and Capital Formation in Latin America", en Luis Servén y Andrés Solimano, Striving for Growth After Adjustment: The Role of Capital Formation, Banco Mundial.

Castillo, Ramón y Jorge Herrera, 2005, “Efecto del gasto público sobre el gasto privado en México", Estudios Económicos, 20:173-196.

Cohen, Daniel, 1993, "Low Investment and Large LDC Debt in the 1980's", American Economic Review, 83: 437-439.

Dickey, D. y W. Fuller, 1979, “Distribution of Estimators for Autoregressive Time Series with a Unit Root", Journal of the American Statistical Association, 74: 427-431.

Elbadawi, Ibrahim, Benno Ndulu y Njuguna, Ndungu, 1997, “Debt Overhang and Economic Growth in Sub-Saharan. Africa", en Zubair Iqhal y Kanbur Ravi (eds.), External Finance for Low Income Countries, Instituto del Fondo Monetario Internacional, Washington, D.C.

Franko, Patrice, 2006, The Puzzle of Latin American Economic Development, Rowman and Littlefield. 
Gavito, M. Javier y Pablo P. Reyes, 1990, “Deuda externa y crecimiento económico: la experiencia reciente de México", Instituto Mexicano de Ejecutivos de Finanzas, México.

Giavazzi, Francesco, Tullio Japelli y Marco Pagano, 2000, “Searching for Non-linear Effects of Fiscal Policy: Evidence from Industrial and Developing Countries", European Economic Review, 44:12591289.

Girón, Alicia, 1991, México, cincuenta años de deuda externa, UNAM, México.

Green, Rosario, 1997, Lecciones de la deuda externa de México, de 1973 a 1997, de abundancias y escaseces, Fondo de Cultura Económica, México.

Hansen, Henrik, 2002, The Impact of Aid and External Debt on Growth and Investment, Credit Research Paper No. 02/26, Centre for Research in Economic Development and International Trade, University of Nottingham.

Herrera, Jorge, 2002, "Dinámica de la inversión privada en México", Gaceta de Economía 15: 225-240.

Lustig, Nora, 1997, "Mexico in Crisis, the US to the Rescue. The Financial Assistance Package of 1982 and 1995", Brookings Discussion Paper.

Rama, Martín, 1993, “Empirical Investment Equations for Developing Countries", en Luis Servén y Andrés Solimano, Striving for Growth After Adjustment: The Role of Capital Formation, Banco Mundial.

Ribeiro, Bruno y Rodolpho Teixeira, 2001, "Análisis econométrico de la inversión privada en Brasil", Revista de la CEPAL 74.

Rubio, M. Orlando, Jair J. Ojeda y Enrique U. Montes, 2003, “Deuda externa, inversión y crecimiento en Colombia (1970-2002)", Banco Central de Colombia.

Secretaría de Hacienda y Crédito Público, 1988, Deuda externa pública mexicana, Fondo de Cultura Económica y Secretaría de Hacienda y Crédito Público, México.

Serieux, John y Samy Yiagadeesen, 2001, “The Debt Service Burden y Growth: Evidence from Low Income Countries", The NorthSouth Institute, Ottawa, Canadá. 
Servén, Luis, y Andrés Solimano, 1993, “Economic Adjustment and Investment Performance in Developing Countries: The Experience of the 1980s", en Luis Servén y Andrés Solimano, Striving for Growth After Adjustment: The Role of Capital Formation, Banco Mundial.

Were, Maureen, 2001, "The Impact of External Debt on Economic Growth and Private Investments in Kenya: An Empirical Assessment", Kenya Institute for Public Policy Research and Analysis. 